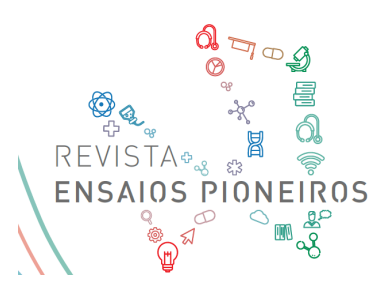

http://ensaiospioneiros.usf.edu.br

\title{
DENTIFRÍCIOS COM DIFERENTES PRINCÍPIOS BIOATIVOS NA REDUÇÃO DA HIPERSENSIBILIDADE DENTINÁRIA CERVICAL: UMA REVISÃO DE LITERATURA
}

DENTIFICATIONS WITH DIFFERENT BIOACTIVE PRINCIPLES IN REDUCING CERVICAL DENTAL HYPERSENSITIVITY: A LITERATURE REVIEW

\author{
ALMEIDA, Marcia Caroline Ovidio Frederico Paes de ${ }^{1}$; CÂMARA, Ana Cristina Zadolynny \\ Raposo da ${ }^{1}$; TOGNETTI, Valdineia $\mathrm{Maria}^{2}$; ANDRADE, Alessandra Pereira de ${ }^{2}$; \\ ${ }^{1}$ Graduandas do Curso de Odontologia - Universidade São Francisco; \\ ${ }^{2}$ Professoras do Curso de Odontologia - Universidade São Francisco.

\section{alessandra.andrade@usf.edu.br}

RESUMO. Atualmente, percebe-se uma alta prevalência de hipersensibilidade dentinária e, com isso, os problemas por ela causados ao indivíduo. Com o avanço tecnológico, muitas têm sido as terapêuticas utilizadas para minimizar seus efeitos, dentre as quais estão os dentifrícios. Sendo assim, o objetivo deste estudo foi avaliar a efetividade de tais dentifrícios e seus diferentes compostos bioativos voltados à redução da hipersensibilidade dentinária dos pacientes. Para isso, o mesmo baseou-se numa revisão de literatura, de abordagem qualitativa e caráter exploratório, tendo por justificativa agregar informações à prática clínica do cirurgião-dentista, principalmente no que se refere à escolha mais assertiva de protocolos que venham auxiliar seu paciente. Os resultados obtidos com a pesquisa apontaram que a terapêutica mais utilizada foi a com base em arginina $8 \%$, embora as demais tenham apresentado mecanismos diferentes, como a Recaldent ${ }^{\mathrm{TM}}$, NovaMin ${ }^{\circledR}$, fluoreto de sódio e nHA. As pesquisas somaram mais de 234 participantes, sendo uma maioria composta de mulheres, com idade entre 18 e 65 anos e um total de mais de 421 dentes hipersensíveis, principalmente de pré-molares. Concluiu-se, com o estudo, que os dentifrícios se mostram eficazes de forma equivalente no que se refere ao tratamento de hipersensibilidade dentinária cervical, embora tenham sido poucos os relatos de casos clínicos que fizeram uso de nHA e Recaldent ${ }^{\mathrm{TM}}$. Obtendo maiores informações em relação aos possíveis tratamentos, é possível proporcionar uma melhor qualidade de vida ao paciente.

Palavras-chave: dentifrícios, hipersensibilidade dentinária cervical, lesão cervical, mineralização dentinária, princípios bioativos.

ABSTRACT. Currently, there is a high prevalence of dentin hypersensitivity and, with this,
the problems caused by it to the individual. With technological advances, many therapies
have been used to minimize its effects, among which are toothpastes. Therefore, the aim of
this study was to evaluate the effectiveness of such dentifrices and their different bioactive
compounds aimed at reducing patients' dentinal hypersensitivity. For this, it was based on a
literature review, with a qualitative approach and an exploratory character, with the
justification of adding information to the clinical practice of the dentist, especially with regard
to the more assertive choice of protocols that come to assist his patient. Os resultados obtidos
com uma pesquisa apontaram que a terapia mais utilizada foi com base em arginina $8 \%$,
embora como demais apresentem diferentes mecanismos, como a Recaldent ${ }^{\mathrm{TM}}$, NovaMin ${ }^{\circledR}$,
fluoreto de sódio e nHA. The surveys totaled more than 234 participants, the majority of
whom were women, aged between 18 and 65 years and a total of more than 421
hypersensitive teeth, mainly premolars. It was concluded, with the study, that dentifrices are 


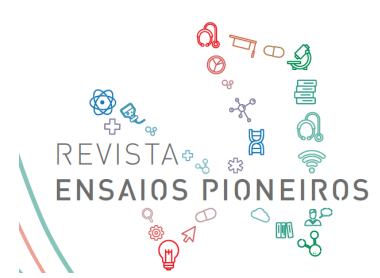

http://ensaiospioneiros.usf.edu.br

equivalent in the treatment of cervical dentin hypersensitivity, although there have been few reports of clinical cases that used $\mathrm{nHA}$ and Recaldent ${ }^{\mathrm{TM}}$. Obtaining more information in relation to possible treatments, it is possible to provide a better quality of life to the patient.

Keywords: dentifrices, cervical dentin hypersensitivity, cervical lesion, dentin mineralization, bioactive principles.

\section{INTRODUÇÃO}

O aumento da expectativa de vida da população mundial, associado a medidas preventivas, acabou por ocasionar o declínio da perda de elementos dentais por patologias bucais, a exemplo da cárie dental e doenças periodontais. Esse aumento no período de permanência dos elementos dentais na cavidade bucal tem possibilitado, por sua vez, uma maior incidência clínica de efeitos deletérios decorrentes do desgaste dental (GUENTSCH et al., 2012).

Esse desgaste é um processo fisiológico natural e tem sido atribuído à tríade erosão, abrasão e atrição. Entretanto, em determinadas circunstâncias e, em alguns indivíduos, o desgaste dental pode se apresentar excessivo e, portanto, patológico. Essa perda patológica de tecido dental mineralizado tem se tornado um problema crescente, não apenas entre pessoas idosas - devido ao aumento da longevidade e da permanência do elemento dental na cavidade bucal -, como também entre adultos e jovens (LIN et al.,2013; ROSA et al., 2013).

Um dos efeitos indesejados mais comumente observado na prática clínica é a Hipersensibilidade Dentinária (HD), que é caracterizada por uma dor curta e aguda decorrente de dentina exposta resultante de vários estímulos, como por exemplo, calor, frio, químicos ou osmóticos que não podem ser atribuídos a qualquer outra forma de defeito ou patologia dental. A teoria hidrodinâmica de Brännström é a explicação mais aceita para esta condição. Com isso, a HD precisa ser abordada para oferecer aos pacientes conforto oral e qualidade de vida (LIN et al., 2013; ROSA et al., 2013).

Nesse contexto, novos produtos têm sido introduzidos no mercado com a proposta de auxiliar na redução da HD, uma vez que atuam como agente dessensibilizante. Um exemplo disso são os dentifrícios, que contêm compostos bioativos, como fosfato de cálcio amorfo e fosfopeptídeo de caseína (CPP-ACP) (Recaldent ${ }^{\mathrm{TM}}$ ), vidro bioativo contendo fosfosilicato de cálcio e sódio (NovaMin $\left.{ }^{\circledR}\right)$ e, ainda, nitrato de potássio e dióxido de titânio. Contudo, há também formulações nas quais esses compostos se apresentam associados a fluoretos (ANAND et al., 2017).

Os agentes bioativos baseados em proteínas do leite, como o complexo CPP-ACP agem pela precipitação de íons cálcio e fosfato, incorporando-os à película adquirida e à placa, atuando como um grande reservatório de íons com potencial remineralizador. Estudos sugerem que o CPP-ACP tem também a capacidade de inibir a desmineralização do esmalte, sendo considerada uma estratégia preventiva importante (CHERMONT et al., 2017; VANO et al., 2014).

Outra tecnologia desenvolvida é a que se baseia em nanopartículas de fosfato de cálcio, as quais têm similaridade com a morfologia dos cristais de hidroxiapatita (HA) e com a estrutura cristalina do esmalte dental. Essa nova pasta apresenta características químicas e estruturais semelhantes à da HA natural. Nos últimos anos, a nano hidroxiapatita (nHA) tem sido amplamente estudada como material biomimético (ANAND et al., 2017; CHERMONT et al., 2017; VANO et al., 2014). 


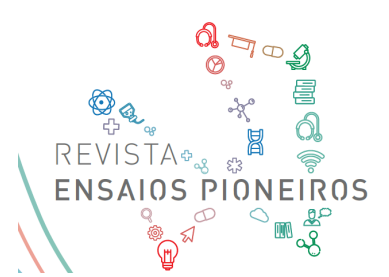

O manejo da HD por meio de oclusão da entrada dos túbulos dentinários por via química e/ou mecânica tem sido relatada como um método eficaz para a redução de sensibilidade. Sendo assim, os dentifrícios são veículos comuns como agentes dessensibilizantes, graças ao seu baixo custo, facilidade de uso e aplicação.

Assim, considerando as altas taxas de prevalência da HD na população mundial, o diagnóstico à eleição de protocolos adequados e efetivos de tratamento é um desafio na clínica odontológica. Nesse sentido, o presente estudo pauta-se na justificativa de agregar informações à prática clínica diária do cirurgião-dentista - por meio de evidências científicas -, principalmente no que se refere à escolha mais assertiva de protocolos que venham auxiliar seu paciente com quadro de HD. A relevância dessa abordagem se dá pelo fato de que os estudos concernentes à temática, especialmente quando por meio da utilização de compostos químicos bioativos, se mostram promissores e relevantes à condução de protocolos adequados e eficientes de tratamento.Portanto, esta pesquisa tem como objetivo avaliar a efetividade dos dentifrícios dentais e seus diferentes compostos bioativos, quando utilizados para reduzir a HD.

\section{METODOLOGIA}

Durante muito tempo o homem deu início a uma jornada em busca do conhecimento, tendo por intuito responder às questões referentes aos seus problemas cotidianos, muitas vezes explicadas pela mitologia. Quando então passou a procurar por explicações mais aceitáveis, tendo por base desta vez a razão, as respostas igualmente se mostraram mais realistas e mais bem aceitas pela sociedade. Com isso, surgia uma ideia inicial de ciência, pela qual se buscava explicar os fenômenos (OLIVEIRA, 2011).

Isso pode ser confirmado pelo fato de que a ciência tem, como principal objetivo, compreender e perceber os fenômenos da natureza, pela qual se busca as respostas que a sociedade necessita. Nesse âmbito, os cientistas compartilham informações entre si para, com isso, obter novos conhecimentos e atualizar os até então obtidos (DROESCHER; SILVA, 2014). Nesse sentido, entende-se que o resultado de uma pesquisa científica deve ser o de contribuir para o avanço do conhecimento humano, sendo sua natureza básica produzir novos conhecimentos que se mostrem úteis à ciência, fazendo-a avançar. Para isso, a verdade e os interesses universais estão envolvidos (PRODANOV; FREITAS, 2013).

O que se entende por metodologia é o estudo sistemático que se realiza, fundamentado pela lógica dos métodos empregados, seus fundamentos, sua validade e, ainda, sua relação com as teorias científicas. Embora os procedimentos variem de uma área a outra, os mesmos procuram descrever o caminho percorrido e os motivos pelos quais foram traçados (OLIVEIRA, 2011). Prova disso é que as pesquisas podem apresentar variados tipos, de acordo com sua natureza, objetivos estabelecidos, procedimentos técnicos que serão utilizados e a maneira com que o problema será tratado durante seu desenvolvimento (PRODANOV; FREITAS, 2013).

No que diz respeito a um dos procedimentos técnicos para realizar uma pesquisa, citase o levantamento de materiais já publicados pertinentes ao assunto que se pretende abordar, em seu desenvolvimento, dentre os quais se encontram os artigos provenientes de revistas científicas, trabalhos acadêmicos para defesa de título, jornais, boletins e até mesmo de entidades que sejam inerentes ao tema, o que a caracteriza como bibliográfica (PRODANOV; FREITAS, 2013). A revisão de literatura é composta pela evolução do tema e ideias advindas de vários autores sobre determinado tema, pela qual são apresentados de maneira dissertativa os conceitos referentes ao assunto do estudo em questão, relacionando-os (OLIVEIRA, 2011). 
A classificação quanto aos objetivos propostos por uma pesquisa, por sua vez, se difere entre descritiva, exploratória, explicativa e exploratório-descritiva (OLIVEIRA, 2011), sendo que na exploratória se levanta materiais para obter maiores dados sobre determinado assunto (PRODANOV; FREITAS, 2013).

A pesquisa pode ser diferenciada também quanto à sua natureza, já que se classifica em qualitativa, quantitativa e qualitativa-quantitativa (OLIVEIRA, 2011). Especificamente quanto à qualitativa, pode-se afirmar que este tipo de abordagem não pode ser mensurado por meio de números, mas fundamenta-se na interpretação das informações colhidas e atribuição de significados (PRODANOV; FREITAS, 2013).

Tendo fundamentado os tipos de pesquisa, pode-se afirmar que este estudo se baseia em uma revisão de literatura, caracterizada como bibliográfica, de natureza qualitativa e com objetivo exploratório.

Contudo, pelo fato de atualmente haver uma gama incontável de assuntos nos meios virtual e físico, foi preciso estabelecer alguns critérios de inclusão ou exclusão daqueles que seriam interessantes ao assunto - ou não. No sentido de restringir a busca somente aos que seriam relevantes, os critérios de inclusão tiveram por base as palavras-chave "dentifrícios", "hipersensibilidade dentinária cervical", "lesão cervical", "mineralização dentinária" e "princípios bioativos", aqueles que foram publicados entre os anos 2010 e 2020 e, ainda, que estivessem nos idiomas português e inglês. Para obter os resultados, foram utilizadas as bases de dados da Biblioteca Virtual em Saúde (BVS), Scientific Eletronic Library Online (SciELO) - traduzida para Biblioteca Eletrônica Científica Online -, PubMed - mantido pela Biblioteca Nacional de Medicina (NLM) dos Estados Unidos da América (EUA) - e Google Acadêmico. Por sua vez, os critérios de exclusão determinados foram aqueles que não estivessem dentro do período supracitado, em outro idioma que não o português e inglês e não continham as palavras-chave estabelecidas.

Durante a busca, foram encontrados 3.390 resultados de variados tipos, dentre os quais cita-se os trabalhos acadêmicos para defesa de título, artigos científicos, de entidades e outros materiais pertinentes. Entretanto, devido à necessidade de uma triagem para considerar aqueles realmente relevantes à discussão, 91 foram mantidos. Justifica-se a exclusão de grande parte desse material colhido pelo fato de que não correspondiam ao assunto em questão, apresentavam vertentes diferentes à pretendida para abordar no presente estudo e que tinham outros princípios bioativos que não os de interesse. Após leitura na íntegra dos que restaram, e considerando os que eram de fato relevantes ao assunto - devido à referência do uso de dentifrícios e seus princípios bioativos, bem como de casos clínicos que viriam a contribuir para a temática -, foram mantidos 39 materiais.

Em relação às obras utilizadas, pode-se afirmar que a maioria ocorreu em território nacional - especificamente na Região Sudeste -, seguida de obras internacionais de várias partes do mundo no idioma inglês. Os anos em que mais houve publicação foram 2012, 2013 e 2018, enquanto uma minoria se deu no de 2010. A maior fonte se deu por meio de revistas científicas, seguidas de sites em geral e de notícias, de trabalhos para defesa de título e, por fim, de livros virtuais.

\section{RESULTADOS E DISCUSSÃO}

\section{Hipersensibilidade da dentinária cervical}

A retração da gengiva faz com que a dentina radicular fique exposta e, gradualmente, haja consequente perda da estrutura mineral do dente, formando uma cavidade - denominada 


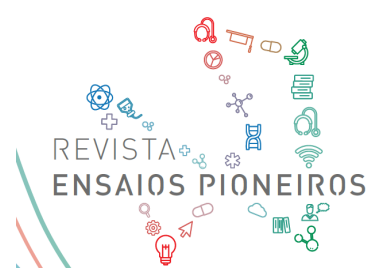

http://ensaiospioneiros.usf.edu.br

de lesão cervical não cariosa (SEBBEN, 2018). Em outras palavras, a dentina fica exposta devido à retração gengival ou por falta de esmalte, motivo que leva à exposição da raiz do dente (R7, 2019).

Por conta de a dentina apresentar um tecido mais poroso se em comparação ao esmalte e possuir microtúbulos, quando estes são estimulados por determinados alimentos ou certas temperaturas, a pessoa apresenta a HD (R7, 2019), também denominada de hipersensibilidade - ou sensibilidade ${ }^{1}$ - da dentina, cervical, da raiz e cimentícia. A variação depende do local em que ocorre (PORTO; ANDRADE; MONTES, 2009; MIGLANI; AGGARWAL; AHUJA, 2010 apud DAVARI; ATAEI; ASSARZADEH, 2013), contudo, os termos carregam consigo a mesma concepção (DAVARI; ATAEI; ASSARZADEH, 2013).

Essa ocorrência pode ser explicada pelo fato do líquido bucal, a placa dentária e o fluído intersticial do mineral conterem cálcio e íons fosfato e, com isso, depende do $\mathrm{pH}$ manter o ambiente dentário saturado, abaixo ou super saturado com relação ao mineral. Em um ambiente insaturado, onde o $\mathrm{pH}$ se encontra inferior a 5,5, ocorre a desmineralização, ao passo que em um ambiente supersaturado ocorre a remineralização, que é quando o pH está acima de 5,5 (AMAECHI; VAN LOVEREN, 2013). Em outras palavras, "[...] as estruturas dentais mineralizadas interagem com os fluídos orais. A [...] [HA] presente no esmalte pode apresentar condição saturada ou não saturada de cálcio e fosfato, oriundos dos fluídos orais, que depende da variação de $\mathrm{pH}$ [...]". Esse processo resulta nos processos denominados de remineralização e desmineralização. Mesmo que a saliva venha a aumentar novamente o $\mathrm{pH}$ e favorecer a reposição de cálcio e fosfato no esmalte, a mesma não ocorre de igual forma e, portanto, há perda mineral reduzida em relação à situação quando em ambiente ácido (MELO et al., 2015, p. 303).

Para Paulo Vinícius Soares - professor, pesquisador da Faculdade de Odontologia da Universidade de Uberlândia e especialista na área -, anteriormente este problema se dava geralmente entre as pessoas com idade superior a 70 anos, mas atualmente pode ser encontrado entre $30 \%$ de jovens com idade entre 25 e 30 anos (TAVARES, 2018). Isso pode ser explicado pelo fato de que o mesmo está presente entre $35 \%$ dos brasileiros, principalmente na faixa dos 30 anos (REVISTA ENCONTRO, 2017), enquanto na população adulta a HD afeta $57 \%$ das pessoas (R7, 2019). Mesmo que a cárie seja o assunto mais abordado na área odontológica, o Brasil possui mais indivíduos com dentes sensíveis se em comparação ao primeiro grupo (BERGAMO, 2018). Prova disso foram as pesquisas realizadas que chegaram a uma prevalência que oscila entre 20 e 50 anos de idade, ao passo que o sexo feminino, aparentemente, é o mais afetado, com certa relação com sua dieta e higiene dental (PORTO; ANDRADE; MONTES, 2009; MIGLANI; AGGARWAL; AHUJA, 2010; ARANHA; PIMENTA; MARCHI, 2009; CHU; LO, 2010; CUMMINS, 2010 apud DAVARI; ATAEI; ASSARZADEH, 2013). No mundo, esse problema afeta $35 \%$ da população (R7, 2019a).

Essas diferenças de prevalência são explicadas pelos divergentes relatos oriundos dos estudos realizados, que consideraram diferentes localidades, hábitos mantidos pelos pacientes, métodos de avaliação, tamanho da amostra considerada (MAIA, 2015), origem étnica, condição periodontal, regime de cuidados dentários, diferentes critérios para determinar o diagnóstico de HD e a fonte de dados que a definiu - se por meio de questionários ou tendo por base a avaliação clínica do paciente (ZADO; PILATTI, 2016). Por vezes, as pesquisas se mostram inclusive escassas - no sentido de determinar uma prevalência mais assertiva do problema -, motivo pelo qual seria preciso realizar estudos epidemiológicos com base em 
amostras representativas. Se dessa maneira, seria possível conhecer melhor este quadro entre a população e os fatores que a ele estão associados (TRENTIN; BERVIAN, 2014).

No que diz respeito aos prejuízos e sintomas da HD, pode-se afirmar que é no inverno que a sensação se mostra mais dolorosa, devido à diferença de temperatura bucal constante entre $36^{\circ}$ e $37^{\circ}$ graus em relação à mudança térmica (R7, 2019a). Um outro problema observado quando da exposição da dentina devido à má escovação, por exemplo, é o incômodo ao beber ou comer algo doce, gelado ou quente (ZIEGLER, 2018), embora possa ocorrer quando na própria escovação (REVISTA ENCONTRO, 2017). Por conta da retração ou recessão gengival, o dente acaba por ficar com um aspecto mais longo. Dependendo do local em que ocorre, isso pode afetar a harmonia e estética do sorriso do indivíduo (SEBBEN, 2018). Ademais, a HD pode levar o paciente a ter problemas psicológicos e físicos, bem como um comprometimento de sua qualidade de vida, principalmente no que diz respeito à seleção de sua dieta (CHU; LO, 2010 apud DAVARI; ATAEI; ASSARZADEH, 2013). Além disso, se não tratada, a Hipersensibilidade Dentinária Cervical (HDC) pode levar a alterações pulpares consideráveis e fraturas dentárias (SANTOS et al., 2010).

São cinco os grupos de risco para ter a HD, sendo que no primeiro se enquadram aqueles que se utilizavam de aparelhos ortodônticos, enquanto no segundo estão aqueles que possuem alguma doença gástrica - como refluxo e úlcera -, se submeteram à cirurgia bariátrica ou que se utilizam de balão intragástrico. $O$ terceiro grupo, por sua vez, é representado por esportistas amadores, que geralmente têm de consumir elevada quantia de bebidas cítricas (TAVARES, 2018). Nos dois últimos grupos, a destruição da camada superficial pode ser explicada devido à alteração do pH bucal $(\mathrm{R} 7,2019)$.

No quarto grupo estão aqueles que provocam uma sobrecarga na articulação temporamandibular, devido à ação de cerrar, ranger e trincar os dentes (TAVARES, 2018), um problema denominado de bruxismo, que pode ocorrer até mesmo durante o sono (R7, 2019).

Por fim, o quinto grupo é representado por pessoas que sofrem de efeitos colaterais de medicamentos ou tratamentos agressivos, a exemplo de radioterapia e quimioterapia (TAVARES, 2018).

Segundo Rayssa Zanatta - professora especialista da Faculdade de Odontologia da Associação Paulista de Cirurgiões-Dentistas (APCD) -, percebe-se que a HD tem causa multifatorial, com uma associação entre fator oclusal, estilo de vida e hábitos alimentares. A abrasão é um desses fatores, que pode ser caracterizada como o desgaste provocado por substâncias presentes em certos cremes dentais (REVISTA ENCONTRO, 2017). Além disso, escovar com força, fazer movimentos inadequados e fazer uso de escovas com cerdas duras também são ações abrasivas que podem levar à sensibilidade (OLIVEIRA, 2020).

A abfração é um tipo de lesão em que o desenvolvimento se dá "[...] quando a carga de oclusão excêntrica leva à flexão de cúspides, originando lesão nas linhas de esmalte na linha gengival, com consequente enfraquecimento da estrutura dentária [...]". A lesão é caracterizada "[...] pela presença de um entalhe cervical profundo em forma de V [...]", embora também possa ser observada quando restaurações na área cervical são realizadas repetidamente (TRENTIN; BERVIAN, 2014, p. 253).

A erosão é outro fator, caracterizada esta como a perda de tecidos duros dentários causados por ácidos não bacterianos. Em associação com ações mecânicas, a exemplo da escovação, resulta-se no desgaste erosivo. A erosão está relacionada à HD pelo fato de que a primeira pode ser um dos fatores que a provoca e até mesmo a mantém (ZIEGLER, 2018).

Ademais, outros fatores que podem levar à sensibilidade dos dentes são a mastigação de objetos (OLIVEIRA, 2020), má oclusão que resulta em microtrincas, quebra de 


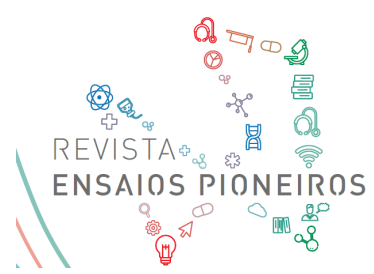

restauração, fratura do dente e clareamento dentário, embora este último cesse gradualmente com o tempo (R7, 2019b).

Dessa maneira, percebe-se que os fatores etiológicos relacionados à HD se dividem em dois tipos de lesões, sendo que num deles há perda de estrutura dental - em que se presencia a erosão, abrasão e abfração -, enquanto outro é aquele em que não há perda de estrutura dental, que são as exposições radiculares (TRENTIN; BERVIAN, 2014).

\section{Terapias para a sensibilidade dentinária cervical}

De acordo com Maia (2015, p. 17), o tratamento da HD se dá por meio da utilização de diversos agentes dessensibilizantes. "[...] Com base no mecanismo de ação, esses agentes podem atuar em dois fatores: na transmissão do impulso nervoso através dos mecanorreceptores e na exposição dos túbulos dentinários". Conforme Zado e Pilatti (2016), os vários tratamentos que a literatura propõe apresentam efetividade, embora com variação em relação ao grau, tempo e efeitos.

Algumas das terapias aplicadas são os géis, enxaguantes bucais, dentifrícios e aqueles de uso tópico, como por exemplo, resina composta, vernizes, cimento de ionômero de vidro, adesivos dentinários, membranas periodontais (GODINHO; GRIPPI; COSTA, 2011), nitrato de potássio, cloreto de estrôncio, fluoretos, oxalatos, lasers, iontoforese, terapia cirúrgica mucogengival de recobrimento radicular, tratamento endodôntico (OLIVEIRA, et al., 2012) e, por fim, os cremes dentais, sendo estes os mais vendidos dentre outros (DAVARI; ATAEI; ASSARZADEH, 2013).

Contudo, ressalta-se que a criação de cremes dentais data de cerca de 3 mil a 5 mil anos atrás, passando por vários incrementos por meio dos egípcios, persas, gregos, romanos, chineses e indianos. Alguns dos problemas comuns em tais cremes eram o custo elevado, gosto ruim e alta abrasividade, motivos pelos quais não eram tão comercializados como atualmente (LIPPERT, 2013). Devido ao aumento da prevalência de erosão e HD, nos últimos anos, foram vários os cremes dentais que foram introduzidos no mercado com diversos atributos. Se há 20 anos eles não existiam, atualmente são várias as marcas que os comercializam no sentido de atender essa demanda (ZIEGLER, 2018). Isso pode ser explicado pelo fato de que entre todas as estratégias criadas até os dias atuais para prevenir os problemas bucais, a escovação dentária é considerada a de melhor impacto. Isso se deve à sua efetividade na remoção ou desestruturação de placa dentária e por ser um método de fácil acesso à população, o que contribuiu para o advento dos dentifrícios, cuja composição com substâncias químicas terapêuticas auxiliam a melhora da saúde bucal do indivíduo (MAGALHÃES et al., 2011).

Prova disso é que, segundo Porto, Andrade e Montes (2009 apud GODINHO; GRIPPI; COSTA, 2011, p. 73), "os cremes dentais são os veículos mais comuns para agentes dessensibilizantes. São amplamente indicados, particularmente devido ao seu baixo custo, facilidade de uso e aplicação caseira [...]". Apresentam fórmulas complexas e vários ingredientes, a exemplo do fluoreto de sódio ( $\mathrm{NaF}$ ), embora também contenham abrasivos como o carbonato de cálcio e fosfato de cálcio - que podem provocar a obliteração dos túbulos quando durante a escovação.

Dentre alguns dentifrícios, alguns contêm fluoreto, os quais, embora tenham surgido na década de 40, foi apenas entre os anos 70 e 80 que houve expansão em sua comercialização, principalmente o que continha sódio - o NaF. O uso de tais dentifrícios "[...] é a forma mais amplamente difundida de autoadministração tópica de fluoretos no mundo" (MAGALHÃES et al., 2011, p. 616). Esse princípio "[...] forma precipitados de cristais de 


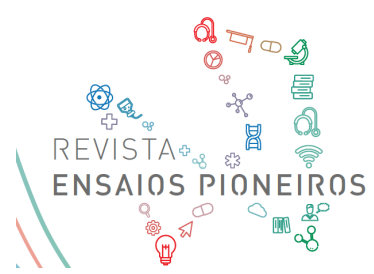

http://ensaiospioneiros.usf.edu.br

flúor dentro dos túbulos dentinários [...]”, fazendo com que haja redução da permeabilidade dentinária e, consequentemente, da sensação dolorosa (DANTAS; MENEZES; DANTAS, 2007; PINTO et al., 2007; DAVARI; ATEI; ASSARZADEH, 2013 apud MAIA, 2015, p. 17). Isso pode ser explicado pelo fato de que o mesmo oblitera os túbulos dentinários, dada a forma de sua atuação: estimula a formação de dentina menos solúvel por conta da reação com os íons de cálcio do fluído dentinário e, dessa maneira, formando os fluoretos de HA (RIBEIRO et al., 2016).

A ação da arginina em um dentifrício, por sua vez, se dá por meio "[...] de uma combinação entre o aminoácido arginina, carbonato de cálcio e o flúor, formando um precipitado que leva a obliteração dos túbulos dentinários" (CUMMINS, 2011; MARQUEZ et al., 2011; DAVARI; ATEI; ASSARZADEH, 2013; HU et al., 2013 apud MAIA, 2015, p. 18). Em outras palavras, o aminoácido carregado positivamente - quando associado ao carbonato de cálcio -, tem a função de se ligar à superfície dentária - negativamente carregada conduzindo a precipitação destes minerais (RIBEIRO et al., 2016).

Mesmo que sob a mesma concepção, a nHA é um princípio bioativo que por vezes diferencia-se entre as siglas HA, HAP, nHAP, nanoHA e nano-HAP -, a qual, segundo Amaechi e Van Loveren (2013, p. 23, tradução livre), é biocompatível e preenche diretamente os microporos nas superfícies desmineralizadas dos dentes. "Quando penetra nos poros do esmalte, também atua como um modelo no processo de remineralização, atraindo continuamente grandes quantidades de íons cálcio e fosfato da solução de remineralização para o tecido do esmalte, promovendo a integridade e o crescimento do cristal". De acordo com Habibah e Salisbury (2020), contudo, a HA já vem sendo utilizada desde a década de 50 na ciência regenerativa. Após 60 anos, por meio de pesquisa e desenvolvimento, a substância vem sendo amplamente oferecida nas áreas da odontologia e medicamentos.

Atualmente, o tratamento da HD também tem se utilizado das propriedades dos vidros bioativos, uma vez que estes "[...] produzem uma resposta biológica específica em suas superfícies quando em contato com os tecidos vivos [...]". Quando na presença de fluídos bucais, os vidros bioativos particulados se depositam no interior dos túbulos dentinários, fazendo com que haja um estímulo à formação de HA e, consequentemente, a oclusão dos túbulos (RIBEIRO et al., 2016, p. 87). Algumas das vantagens do fosfosilicato de cálcio e sódio (NovaMin ${ }^{\circledR}$ ) a $15 \%$ - quando em pasta profilática -, por exemplo, são seu baixo custo e fácil utilização pelo clínico (SILVA, 2018).

A substância Amorphous Calcium Phosphate (ACP) - traduzida para fosfato de cálcio amorfo - vem sendo comercializada como redutora de HD, com aplicação frequente. A longo prazo, a substância pode promover a formação de cálculo dentário, embora essa ocorrência ainda deva ser comprovada por meio de estudos (AMAECHI; VAN LOVEREN, 2013). Quando o ACP se liga ao fosfopeptídeo caseína, ambos formam o complexo CPP-ACP (Recaldent ${ }^{\mathrm{TM}}$ ), sendo que o ACP “[...] é capaz de liberar íons de cálcio e fosfato, mantendo um estado supersaturado, otimizando o processo de remineralização" (REYNOLDS, 1998; TANTBIROJN et al., 2008; ADEBAYO; BURROW; TYAS, 2008 apud MELO et al., 2015, p. 303). Seu mecanismo de ação pode ser explicado pela obliteração dos túbulos dentinários expostos, auxiliando, com isso, a remineralização das estruturas dentárias (RIBEIRO et al., 2016). Uma das vantagens do ACP "[...] é a sua biodisponibilidade, mesmo em pacientes com baixo fluxo salivar, pois o ACP pode se ligar a fluoretos, aumentando assim a captação de flúor e formando uma camada de apatita protetora, na qual os cristais são maiores e mais resistentes a desmineralização" (YAMAMOTO, 2012, p. 19).

Percebe-se que o tratamento da HD se divide entre dois métodos: os que obliteram os túbulos dentinários e os que atuam sobre a propagação dos impulsos nervosos, que se dá por 


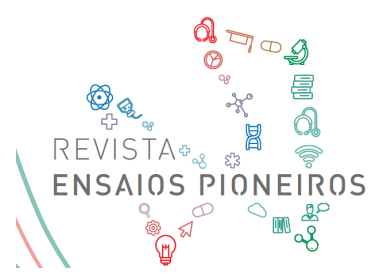

http://ensaiospioneiros.usf.edu.br

meio da iniciação e/ou condução (RIBEIRO et al., 2016). De qualquer forma, estudos confirmaram que algumas das pastas analisadas não se mostraram suficientes para evitar a perda da estrutura do esmalte. Dessa forma, evidenciou-se que devem ser utilizadas como complemento, e não como tratamento (ZIEGLER, 2018).

Godinho, Grippi e Costa (2011) realizaram uma pesquisa junto a 29 pacientes com sintomas de sensibilidade dental, no sentido de verificar a eficácia de cremes dentais: um contendo como ingredientes ativos o carbonato de cálcio, arginina 8\% e monofluorfosfato de sódio $1,10 \%$, e outro contendo acetato de estrôncio $8 \%$, carbonato de cálcio e $\mathrm{NaF}$. Os participantes tinham idade entre 21 e 63 anos, com uma média de 42,6 anos, sendo que 24 eram do sexo feminino e apenas cinco do sexo masculino. Ao todo foram 86 os dentes estudados, compostos por $54,65 \%$ de pré-molares, $23,25 \%$ de molares, $11,63 \%$ de caninos e $10,47 \%$ de incisivos. Após avaliação inicial de cada um deles, os mesmos foram divididos em dois grupos de acordo com a aplicação dos dois dentifrícios escolhidos. Periodicamente era feito um teste para autoavaliação por parte do paciente em relação ao desconforto que sentia: antes e após a aplicação do dentifrício. A redução de dor foi significativa para ambos os grupos e quase semelhantes entre si, com médias dos níveis de redução da dor em aproximadamente 2,3 e 2,84, respectivamente. O que se concluiu, com o estudo, é que a arginina e carbonato de cálcio aliviaram de imediato a sensação de dor sentida pelo paciente.

Maia (2015) realizou um estudo parecido, contudo, utilizando-se como comparativo uma solução de $\mathrm{NaF}$ a $4 \%$ e arginina $8 \%$. O objetivo foi avaliar o grau de sensibilidade após a aplicação do dentifrício, de acordo com o número de aplicações e a efetividade de ambas. A amostra foi composta de 104 dentes - com uma prevalência de 47 pré-molares, 28 molares, 16 caninos e 13 incisivos -, a partir de 13 pacientes com idade a partir de 14 anos, sendo separados em dois grupos: o primeiro recebeu a aplicação de $\mathrm{NaF}$ a $4 \%$, enquanto ao segundo grupo foi aplicada a arginina $8 \%$. Nas sessões que foram realizadas houve avaliação para verificação da redução da dor e, por fim, chegou-se à conclusão de que ambos os tratamentos mostraram efetividade ao apresentar redução significativa da dor, embora não discrepantes a ponto de determinar qual deles de fato é superior. Ambas as substâncias mostraram eficácia, de baixo custo de aplicação e tornaram possível contribuir positivamente com a qualidade de vida do paciente com HD, uma vez que os transtornos por ela causados foram minimizados.

No sentido de avaliar comparativamente a eficácia de duas substâncias, Alves (2019) realizou um estudo tendo por base uma solução de $\mathrm{NaF} 4 \%$ e laser de baixa potência para pacientes com HDC, os quais eram atendidos nas Clínicas Integradas do Departamento de Odontologia da Universidade Federal do Rio Grande do Norte (UFRN). Os 21 participantes incluídos tinham idade superior a 18 anos, embora apenas 13 tenham concluído o tratamento, dos quais quatro eram homens e nove eram mulheres. Ao todo, foram 94 elementos dentários considerados, dos quais $46,80 \%$ eram pré-molares, $28,72 \%$ eram molares, $12,76 \%$ eram incisivos e $11,70 \%$ caninos. A redução da sensibilidade do grupo 1 - para o qual foi aplicado o fluoreto - se mostrou significativa durante e após o tratamento. No que diz respeito à comparação com a outra técnica, não houve diferença considerável, mas pode-se afirmar que o fluoreto se apresenta uma alternativa eficaz e de baixo custo.

Um estudo semelhante foi realizado a partir de 70 pacientes com quadro de HD. De ambos os sexos, as pessoas incluídas tinham idade entre 18 e 65 anos. Os participantes foram divididos em três grupos de maneira aleatória, sendo que em um deles 23 se sujeitaram à aplicação da pasta profilática NovaMin ${ }^{\circledR}$. Destes, 17 estavam com dor severa e 6 com moderada. Após o tratamento, os mesmos retornaram para avaliação. Com o estudo, chegouse à conclusão de que houve redução na dor - durante e após o tratamento -, e entre os demais 


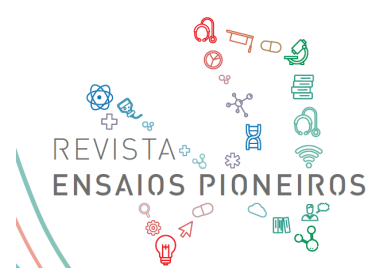

dois grupos considerados com outras aplicações, não houve diferença significativa, ou seja, não foi possível determinar qual deles apresentou maior queda de dor (SILVA, 2018).

Rajesh et al. (2012) realizaram um estudo que durou 11 meses, no qual foram avaliados 30 pacientes com idade entre 18 e 65 anos com quadro de HD. Os participantes foram divididos igualmente em dois grupos: um sujeito à aplicação de creme dental contendo fosfosilicato de sódio a $5 \%$ de cálcio e outro sujeito ao uso de um creme desprovido de agente dessensibilizante. O primeiro grupo, que fez uso do dentifrício NovaMin ${ }^{\circledR}$ foi o que mostrou melhor resultado em relação à redução da sensibilidade, em comparação ao grupo do placebo.

Também Amaechi et al. (2018) realizaram um estudo comparativo, fazendo-se uso de um creme dental contendo $20 \%$ de nHAP com outra substância, tendo por base uma maioria de pacientes oriunda do Centro de Ciências da Saúde da Universidade do Texas, na Faculdade de Odontologia de San Antonio (UTHSCSA). Com idade entre 18 e 80 anos, foram 51 os participantes desta pesquisa, sendo 41 mulheres e 10 homens, com média de idade de 45,5 anos. O grupo que participou da aplicação de nHAP foi composto de 25 indivíduos e, diante dos resultados, pode-se afirmar que o mesmo se mostrou eficaz.

Estudo parecido foi o realizado por Wang et al. (2016), no qual 28 pessoas participaram, sendo sete homens e 21 mulheres com idade entre 18 e 60 anos. As mesmas eram atendidas pela Faculdade de Odontologia de Bauru, das quais somaram 137 dentes hipersensíveis. Com uma quantia de 6 a 8 pessoas por grupo, a um deles foi aplicada a nHA com associação com nitrato de potássio, ao passo que em outro foi aplicada uma pasta profissional e creme dental - ambos com arginina $8 \%$ e carbonato de cálcio -, mas o creme ainda possuía, adicionalmente, monofluorfosfato de sódio e fluoreto. Este último grupo foi o único que mostrou redução significativa - e progressiva - durante o tratamento e, no que se refere aos demais grupos, não houve diferença.

Por fim, em um estudo mais abrangente, considerou-se o NaF e o fosfopeptídeo de caseína-fosfato de cálcio amorfo fluoretado (CPP-ACPF), além de outros dois agentes tópicos para efeitos comparativos de sua eficácia, com a participação de pessoas com idade entre 20 e 40 anos. Diante da pesquisa, chegou-se à conclusão de que o grupo sujeito à aplicação de $\mathrm{NaF}$ apresentou maior eficácia se em relação ao grupo que ficou sujeito ao CPP-ACPF (MADHAVAN et al., 2012).

Diante dos 8 casos clínicos de pacientes com quadro de HD apresentados, conclui-se que os mesmos somaram mais de 421 dentes hipersensíveis e mais de 234 participantes. A inexatidão dessas informações se deve ao fato de que alguns estudos não informaram a quantia de dentes estudados, o gênero dos participantes ou quantos deles os compuseram.

Em relação ao gênero dos participantes, uma maioria foi composta de mulheres (GODINHO; GRIPPI; COSTA, 2011; WANG et al., 2016; AMAECHI et al., 2018; ALVES, 2019), enquanto um dos estudos apenas mencionou que considerou pacientes de ambos os sexos (SILVA, 2018). Essa grande parte da amostra representada pelas mulheres é confirmada pela literatura, considerando outras pesquisas que foram realizadas para determinar a prevalência da HD (SILVA, et al., 2011; ÇOLAK et al., 2012).

No que se refere aos estudos que mencionaram a idade dos participantes, pode-se afirmar que a maioria apontou uma faixa etária entre 18 e 65 anos (GODINHO; GRIPPI; COSTA, 2011; MADHAVAN et al., 2012; RAJESH et al., 2012; WANG et al., 2016; SILVA, 2018), seguido de uma minoria com idade entre 18 e 80 anos (AMAECHI et al., 2018), além de outra pesquisa que considerou pacientes com a partir de 14 anos (MAIA, 2015; ALVES, 2019). A média de idade dos pacientes - quando mencionada no estudo - foi de 40 a 50 anos (GODINHO; GRIPPI; COSTA, 2011; AMAECHI et al., 2018). O intervalo 


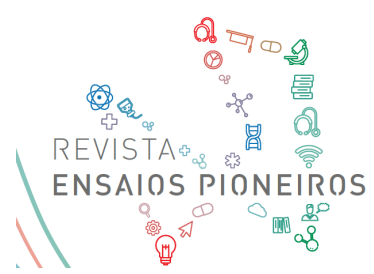

http://ensaiospioneiros.usf.edu.br

de idade supracitado foi um dado que se confirmou, junto à literatura, já que em outras pesquisas a variação se deu entre 18 e 69 anos (ÇOLAK et al., 2012; SILVA et al., 2011).

No que diz respeito aos dentes hipersensíveis considerados, os estudos se referiram em grande parte aos pré-molares como os mais afetados (GODINHO; GRIPPI; COSTA, 2011; MAIA, 2015; ALVES, 2019), e isso se confirma com outros que apontaram os pré-molares como os mais acometidos (SILVA et al., 2011; ÇOLAK et al., 2012).

Pesquisas realizadas apontaram fatores que podem ter contribuído para o quadro de HD entre os pacientes estudados, dentre os quais cita-se os hábitos de alimentação - ao ingerir bebidas geladas e quentes -, de escovação, de tabagismo (ÇOLAK et al., 2012), de ingestão de bebidas ácidas e, ainda, por conta de problema gastrointestinal (SILVA et al., 2011).

Dentre os princípios bioativos mais citados encontra-se a arginina 8\% (GODINHO; GRIPPI; COSTA, 2011; MAIA, 2015; WANG et al., 2016), seguida igualmente pelo NaF (MADHAVAN et al., 2012; MAIA, 2015; ALVES, 2019) e do NovaMin ${ }^{\circledR}$ (RAJESH et al., 2012; SILVA, 2018). O nHAP foi utilizado com associações de substâncias (WANG et al., 2016; AMAECHI et al., 2018), enquanto o CPP-ACPF foi o que menos foi aplicado (MADHAVAN et al., 2012). Entretanto, pode-se afirmar que todas aliviaram a sensação de dor sentida pelos pacientes em diferentes níveis.

Um dos estudos relatou que o $\mathrm{NaF}$ apresentou maior eficácia em relação ao CPPACPF (MADHAVAN et al., 2012), enquanto um outro não conseguiu confirmar qual das terapêuticas aplicadas foi a mais eficaz se em comparação à outra, por motivos de equivalência entre elas (MAIA, 2015; WANG et al., 2016; SILVA, 2018; ALVES, 2019).

Percebe-se que, no sentido de prover um controle da dor relatada pelo paciente e evolução das lesões que a ela dão origem, isso representa um desafio ao cirurgião-dentista da atualidade (SILVA, 2018), motivo pelo qual estes profissionais continuam à busca de tratamentos mais eficazes para pacientes com HD (GODINHO; GRIPPI; COSTA, 2011). Isso pode ser comprovado pelo fato da HD se mostrar uma condição frequente, bem como pelo tratamento apresentar dificuldades concernentes à efetividade e durabilidade, uma vez que em alguns casos há recidiva de dor de maneira precoce (ZADO; PILATTI, 2016).

No que se refere às responsabilidades do tratamento, deve o paciente realizar visitas e acompanhamentos junto ao dentista após a realização de um tratamento (DAVARI; ATAEI; ASSARZADEH, 2013), enquanto ao profissional cabe orientar o paciente para que este se utilize de pastas dessensibilizantes, escovar adequadamente seus dentes, fazer uso de instrumentos devidos para higienização bucal, alterar sua dieta e, se o caso, indicar um tratamento. Com essas ações, é possível reduzir o desconforto sentido pelo indivíduo em relação aos fatores que o causam ou potencializam-no, bem como prevenindo o surgimento de lesões ou progressões das já existentes (SILVA et al., 2011).

\section{CONCLUSÃO}

A boca é uma das mais importantes partes do corpo humano, pois é por onde o indivíduo se alimenta e se comunica. Quando com quadro de HD, os sintomas podem prejudicar sua qualidade de vida e levar a problemas graves. Ao verificar seu diagnóstico junto a um profissional, o paciente deve se utilizar de medidas que minimizem seus efeitos.

Um exemplo de terapêutica é a que se utiliza de princípios bioativos em dentifrícios, que podem ser aplicados segundo as orientações do profissional. Dentre vários deles, cita-se o Recaldent ${ }^{\mathrm{TM}}$, NovaMin ${ }^{\circledR}, \mathrm{NaF}, \mathrm{nHA}$ e arginina, dos quais, diante da pesquisa, foi possível concluir que todos se mostraram eficazes. 
Tendo esta pesquisa o objetivo de avaliar a efetividade dos dentifrícios dentais e seus diferentes compostos bioativos, pode-se afirmar que atingiu o proposto, contudo, com limitações, já que mesmo diante de uma extensa busca na literatura, pouco se encontrou sobre as terapêuticas que se utilizam de HA e CPP-ACP, motivo pelo qual foi preciso ampliar a busca nas bases de dados. Prova disso foi que, dentre os casos citados, grande parte se voltou ao uso da arginina $8 \%$, NovaMin ${ }^{\circledR}$ e $\mathrm{NaF}$.

Com isso, uma sugestão de trabalho futuro seria considerar a busca somente pelas terapêuticas voltadas à HA e CPP-ACP, no sentido de explorar sua eficácia em comparação às demais. Outra sugestão para continuar este estudo seria realizar uma pesquisa de campo, na qual se verificaria quais são os dentifrícios utilizados pelos cirurgiões-dentistas junto a seus pacientes, tendo por intuito conhecer a prevalência e os tratamentos sugeridos. A justificativa para a realização desse estudo mais aprofundado tem por base o já colocado na literatura, na qual se menciona a falta de padronização nos dados referentes à amostra, a exemplo do gênero do indivíduo, sua faixa etária, média de idade, dentes hipersensíveis - e quais deles o são -, hábitos alimentares e estilo de vida. Na presente pesquisa, por exemplo, os dados chegaram a mais de 421 dentes hipersensíveis estudados - em especial os pré-molares -, com mais de 234 participantes, sendo uma maioria composta de mulheres e com idade entre 18 e 65 anos, contudo, faltaram detalhes importantes no que diz respeito à amostra, implicando numa prevalência pouco assertiva.

Portanto, ainda se faz preciso fundamentar bem a amostra participante em um estudo, pois o estilo de vida, hábitos alimentares, gênero e idade podem contribuir para um resultado positivo - ou não - no tratamento, motivo pelo qual ainda é necessário que os profissionais da área busquem mais informações e complementem as até então existentes, no sentido de, com isso, prover melhor qualidade de vida ao paciente.

\section{REFERÊNCIAS}

ALVES, K. R. L. Comparação da eficácia do fluoreto de sódio a 4\% e do laser AsGaAl no tratamento da hipersensibilidade dentinária cervical. 2019. 59 f. Dissertação (Mestrado em Saúde Coletiva) - Universidade Federal do Rio Grande do Norte, Natal, 2019.

AMAECHI, B. T. et al. Clinical Efficacy in Relieving Dentin Hypersensitivity of Nanohydroxyapatite-containing Cream: A Randomized Controlled Trial. The Open Dentistry Journal, v. 12, p. 572-585, 2018.

AMAECHI, B. T.; VAN LOVEREN, C. Fluorides and Non-Fluoride Remineralization Systems. Monographs in Oral Science, [Basiléia], v. 23, p. 15-26, 2013.

ANAND, S. et al. Comparative Evaluation of Effect of Nano-hydroxyapatite and 8\% Arginine Containing Toothpastes in Managing Dentin Hypersensitivity: Double Blind Randomized Clinical Trial. Acta Medica (Hradec Kralove), [s.1.], v. 60, n. 3, p. 114-119, 2017.

BERGAMO, K. Sensibilidade nos dentes: como prevenir ou acabar com ela. Veja, Saúde, [São Paulo], 29 jan. 2018. Disponível em: $<$ https://saude.abril.com.br/medicina/sensibilidadenos-dentes-como-prevenir-ou-acabar-com-ela/>. Acesso em: 3 jul. 2020. 
CHERMONT, A. B. et al. O uso prévio de dessensibilizantes interfere na resistência de união de sistemas adesivos à dentina? Revista da Associação Paulista de CirurgiõesDentistas, São Paulo, v. 71, n. 4, p. 406-412, 2017.

ÇOLAK, $\mathrm{H}$. et al. Prevalence of dentine hypersensitivity among adult patients attending a dental hospital clinic in Turkey. West Indian Medical Journal, Mona, v. 61, n. 2, p. 174179, mar. 2012.

DAVARI, A. R.; ATAEI, E.; ASSARZADEH, H. Dentin Hypersensitivity: Etiology, Diagnosis and Treatment; A Literature Review. Journal of Dentistry, [Shiraz], v. 14, n. 3, p. 136-145, set. 2013.

DROESCHER, F. D.; SILVA, E. L. O pesquisador e a produção científica. Perspectivas em Ciência da Informação, Belo Horizonte, v. 19, n. 1, p. 170-189, jan./mar. 2014. Disponível em: $<$ http://www.scielo.br/scielo.php?script=sci_arttext\&pid=S1413-99362014000100011>. Acesso em: 28 jun. 2020.

GODINHO, C. J.; GRIPPI, M. F.; COSTA, L. C. Avaliação clínica do uso de dois novos cremes dentais no tratamento da hipersensibilidade dentinária. Revista de PósGraduação, São Paulo, v. 18, n. 2, p. 72-78, abr./jun. 2011.

GUENTSCH, A. et al. Biomimetic mineralization: long-term observations in patients with dentin sensitivity. Dental Materials, [s.1.], v. 28, n. 4, p. 457-464, abr. 2012.

HABIBAH, T. U.; SALISBURY, H. G. Hydroxyapatite Dental Material. In: StatPearls. Treasure Island: StatPearls Publishing, 2020.

LIN, P. Y. et al. In-office treatment for dentin hypersensitivity: a systematic review and network meta-analysis. Journal of Clinical Periodontology, [s.1.], v. 40, p. 53-64, jan. 2013.

LIPPERT, F. An Introduction to Toothpaste - Its Purpose, History and Ingredients. Monographs in Oral Science, [Basiléia], v. 23, p. 1-14, 2013.

MADHAVAN, S. et al. Dentinal hypersensitivity: A comparative clinical evaluation of CPP-ACP F, sodium fluoride, propolis, and placebo. Journal of Conservative Dentistry, [Mumbai], v. 15, n. 4, p. 315-318, out.-dez. 2012.

MAGALHÃES, A. C. et al. Uso racional dos dentifrícios. Revista Gaúcha de Odontologia, Porto Alegre, v. 59, n. 4, p. 615-625, out./dez. 2011.

MAIA, B. R. Estudo comparativo da utilização de uma solução de fluoreto de sódio a $4,0 \%$ e de um dentifrício contendo $8,0 \%$ de arginina para o tratamento da hipersensibilidade dentinária cervical. 2015. 24 f. Trabalho de Conclusão de Curso (Graduação em Odontologia) - Universidade Federal do Rio Grande do Norte, Natal, 2015.

MELO, L. F. B. et al. Efeito remineralizador de caseína fosfato de cálcio fosfopeptídeo amorfo em dentes decíduos e permanentes in vivo. Revista da Associação Paulista de Cirurgiões-Dentistas, [São Paulo], v. 69, n. 3, p. 302-306, 2015. 
OLIVEIRA, J. M. et al. Hipersensibilidade dentinária: considerações para o sucesso em seu manejo clínico. HU Revista, Juiz de Fora, v. 38, n. 1-2, p. 13-20, jan./jun. 2012.

OLIVEIRA, M. F. Metodologia científica: um manual para a realização de pesquisas em Administração. Catalão: UFG, 2011. 72 p. :

OLIVEIRA, S. 10 coisas que afetam os dentes e talvez você nem imagine. Uol, Viva Bem, [s.1.], 28 fev. 2020. Disponível em:

$<$ https://www.uol.com.br/vivabem/noticias/redacao/2020/02/28/10-coisas-que-afetam-osdentes-e-talvez-voce-nem-imagine.htm>. Acesso em: 3 jul. 2020.

PRODANOV, C. C.; FREITAS, E. C. Metodologia do trabalho científico: métodos e técnicas da pesquisa e do trabalho acadêmico. 2. ed. Novo Hamburgo: Feevale, 2013. 276 p.

R7. Dores e sensibilidade nos dentes: Por que são mais comuns no inverno? R7, Folha Vitória, [Vitória], 4 abr. 2019. Disponível em:

$<$ https://www.folhavitoria.com.br/saude/noticia/04/2019/dores-e-sensibilidade-nos-dentespor-que-sao-mais-comuns-no-inverno $>$. Acesso em: 3 jul. 2020.

R7. Saiba como lidar com a sensibilidade dos dentes. R7, Folha Vitória, [Vitória], 28 nov. 2019. Disponível em: <https://www.folhavitoria.com.br/saude/noticia/11/2019/saiba-comolidar-com-a-sensibilidade-dos-dentes $>$. Acesso em: 3 jul. 2020.

RAJESH, K. S. et al. Evaluation of the efficacy of a 5\% calcium sodium phosphosilicate (Novamin ${ }^{\circledR}$ ) containing dentifrice for the relief of dentinal hypersensitivity: A clinical study. Indian Journal of Dental Research, [Mumbai], v. 23, n. 3, p. 363-367, 2012.

REVISTA ENCONTRO. Dentes sensíveis têm várias causas, incluindo problemas na mastigação. Revista Encontro, Atualidades, [Belo Horizonte], 7 ago. 2017. Disponível em: $<$ https://www.revistaencontro.com.br/canal/atualidades/2017/08/dentes-sensiveis-tem-variascausas-incluindo-problemas-na-mastigacao.html>. Acesso em: 2 jul. 2020.

RIBEIRO, P. J. T. et al. Mecanismos de ação dos recursos terapêuticos disponíveis para o tratamento da hipersensibilidade dentinária cervical. Odontologia Clínico-Científica, Recife, v. 15, n. 2, p. 83-90, abr./jun. 2016.

ROSA, W. L. O. et al. The effectiveness of current dentin desensitizing agents used to treat dental hypersensitivity: a systematic review. Quintessence International, [Berlim], v. 44, n. 7, p. 535-546, jul. 2013.

SANTOS, A. P. M. et al. Um sintoma preocupante: a hipersensibilidade dentinária. Revista Brasileira de Odontologia, Rio de Janeiro, v. 67, n. 2, p. 242-246, jul./dez. 2010.

SEBBEN, K. Você sofre com retração gengival? Diário do Sudoeste, [Pato Branco], 21 set. 2018. Disponível em: <https://www.diariodosudoeste.com.br/noticia/voce-sofre-comretracao-gengival>. Acesso em: 2 jul. 2020. 
SILVA, B. S. et al. Ocorrência da hipersensibilidade dentinária e seus fatores de risco. Revista de Cirurgia e Traumatologia Buco-Maxilo-Facial, Camaragibe, v. 11, n. 1, p. 99-105, jan./mar. 2011.

SILVA, V. M. Laser de Nd:YAG e pasta profilática de fosfosilicato de cálcio e sódio no tratamento da hipersensibilidade dentinária cervical: um estudo clínico randomizado duplo-cego. 2018. 106 f. Dissertação (Mestrado em Ciências) - Faculdade de Odontologia, Universidade de São Paulo, São Paulo, 2018.

TAVARES, M. Especialista alerta para risco de envelhecimento precoce dos dentes. G1, Bem Estar, Rio de Janeiro, 30 ago. 2018. Disponível em:

$<$ https://g1.globo.com/bemestar/blog/longevidade-modo-de-

usar/post/2018/08/30/especialista-alerta-para-risco-de-envelhecimento-precoce-dosdentes.ghtml>. Acesso em: 2 jul. 2020.

TRENTIN, M. S.; BERVIAN, J. Hipersensibilidade dentinária cervical: uma revisão de literatura. Revista da Faculdade de Odontologia, Passo Fundo, v. 19, n. 2, p. 252-257, maio/ago. 2014.

VANO, M. et al. Effectiveness of nano-hydroxyapatite toothpaste in reducing dentin hypersensitivity: a double-blind randomized controlled trial. Quintessence International, [Berlim], v. 45, n. 8, p. 703-711, set. 2014.

WANG, L. et al. Treatment of Dentin Hypersensitivity Using Nano-Hydroxyapatite

Pastes: A Randomized Three-Month Clinical Trial. Operative Dentistry, [s.1.], v. 41, n. 4, e93-e101, 2016.

YAMAMOTO, T. W. Efeito da utilização de dentifrícios com diferentes compostos bioativos nas propriedades superficiais do esmalte dental clareado. 2012. $55 \mathrm{f}$.

Dissertação (Mestrado em Odontologia) - Faculdade de Odontologia, Universidade de São Paulo, São Paulo, 2012.

ZADO, L. N.; PILATTI, G. L. Hipersensibilidade dentinária: recentes avanços e tratamentos - revisão de literatura. Revista Periodontia, [Belo Horizonte], v. 26, n. 2, p. 28-33, jun. 2016.

ZIEGLER, M. F. Cremes dentais não resolvem sozinhos erosão dental e hipersensibilidade dos dentes. Agência FAPESP, [São Paulo], 9 fev. 2018. Disponível em: $<$ http://agencia.fapesp.br/cremes-dentais-nao-resolvem-sozinhos-erosao-dental-ehipersensibilidade-dos-dentes/27117/>. Acesso em: 2 jul. 2020.

Publicado em 18/08/2021 